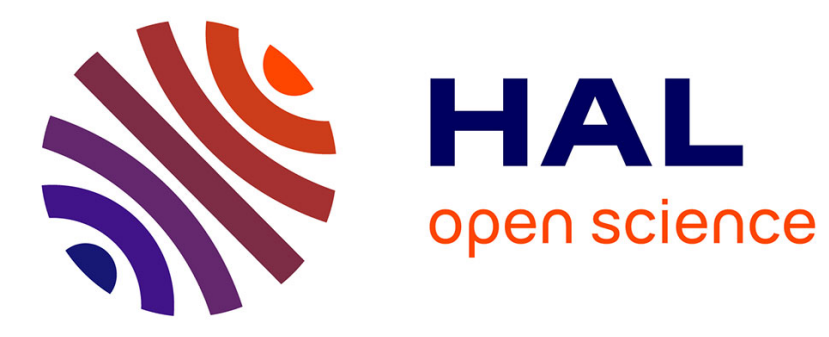

\title{
Development of a bedside score to predict dengue severity
}

\author{
Ingrid Marois, Carole Forfait-Dubuc, Catherine Inizan, Elise Klement-Frutos, \\ Anabelle Valiame, Daina Aubert, Ann-Claire Gourinat, Sylvie Laumond, \\ Emilie Barsac, Jean-Paul Grangeon, et al.
}

\section{To cite this version:}

Ingrid Marois, Carole Forfait-Dubuc, Catherine Inizan, Elise Klement-Frutos, Anabelle Valiame, et al.. Development of a bedside score to predict dengue severity. BMC Infectious Diseases, 2021, 21 (1), pp.470. 10.1186/s12879-021-06146-z . hal-03237167

\section{HAL Id: hal-03237167 \\ https://hal.sorbonne-universite.fr/hal-03237167}

Submitted on 26 May 2021

HAL is a multi-disciplinary open access archive for the deposit and dissemination of scientific research documents, whether they are published or not. The documents may come from teaching and research institutions in France or abroad, or from public or private research centers.
L'archive ouverte pluridisciplinaire HAL, est destinée au dépôt et à la diffusion de documents scientifiques de niveau recherche, publiés ou non, émanant des établissements d'enseignement et de recherche français ou étrangers, des laboratoires publics ou privés.

\section{(c)(1)}

Distributed under a Creative Commons Attribution| 4.0 International License 


\section{Development of a bedside score to predict dengue severity}

Ingrid Marois ${ }^{1+}$, Carole Forfait ${ }^{2 \dagger}$, Catherine Inizan ${ }^{3 \dagger}$, Elise Klement-Frutos ${ }^{1,4^{*}+} \mathbb{D}$, Anabelle Valiame $^{2}$, Daina Aubert ${ }^{2}$, Ann-Claire Gourinat ${ }^{5}$, Sylvie Laumond ${ }^{2}$, Emilie Barsac ${ }^{5}$, Jean-Paul Grangeon², Cécile Cazorla', Audrey Merlet ${ }^{1}$, Arnaud Tarantola ${ }^{6}$, Myrielle Dupont-Rouzeyrol ${ }^{3}$ and Elodie Descloux ${ }^{1+^{\wedge}}$

\section{Abstract}

Background: In 2017, New Caledonia experienced an outbreak of severe dengue causing high hospital burden (4379 cases, 416 hospital admissions, 15 deaths). We decided to build a local operational model predictive of dengue severity, which was needed to ease the healthcare circuit.

Methods: We retrospectively analyzed clinical and biological parameters associated with severe dengue in the cohort of patients hospitalized at the Territorial Hospital between January and July 2017 with confirmed dengue, in order to elaborate a comprehensive patient's score. Patients were compared in univariate and multivariate analyses. Predictive models for severity were built using a descending step-wise method.

Results: Out of 383 included patients, 130 (34\%) developed severe dengue and 13 (3.4\%) died. Major risk factors identified in univariate analysis were: age, comorbidities, presence of at least one alert sign, platelets count $<30 \times$ $10^{9} / \mathrm{L}$, prothrombin time $<60 \%$, AST and/or ALT $>10 \mathrm{~N}$, and previous dengue infection. Severity was not influenced by the infecting dengue serotype nor by previous Zika infection.

Two models to predict dengue severity were built according to sex. Best models for females and males had respectively a median Area Under the Curve $=0.80$ and 0.88 , a sensitivity $=84.5$ and $84.5 \%$, a specificity $=78.6$ and $95.5 \%$, a positive predictive value $=63.3$ and $92.9 \%$, a negative predictive value $=92.8$ and $91.3 \%$. Models were secondarily validated on 130 patients hospitalized for dengue in 2018.

Conclusion: We built robust and efficient models to calculate a bedside score able to predict dengue severity in our setting. We propose the spreadsheet for dengue severity score calculations to health practitioners facing dengue outbreaks of enhanced severity in order to improve patients' medical management and hospitalization flow.

Keywords: Dengue, Arboviruses, Severity score, Operational tool, Hospital triage, Pacific

\footnotetext{
* Correspondence: elisemma@hotmail.com

Elodie Descloux is deceased.

${ }^{\dagger}$ Ingrid Marois, Carole Forfait, Catherine Inizan, Elise Klement-Frutos and

Elodie Descloux contributed equally to this work.

${ }^{1}$ Internal Medicine and Infectious Diseases Department, Territorial Hospital

Center (CHT), Dumbea, New Caledonia

${ }^{4}$ Hôpitaux Universitaires Pitie Salpetriere-Charles Foix, Paris, France

Full list of author information is available at the end of the article
}

C C The Author(s). 2021 Open Access This article is licensed under a Creative Commons Attribution 4.0 International License, which permits use, sharing, adaptation, distribution and reproduction in any medium or format, as long as you give appropriate credit to the original author(s) and the source, provide a link to the Creative Commons licence, and indicate if changes were made. The images or other third party material in this article are included in the article's Creative Commons licence, unless indicated otherwise in a credit line to the material. If material is not included in the article's Creative Commons licence and your intended use is not permitted by statutory regulation or exceeds the permitted use, you will need to obtain permission directly from the copyright holder. To view a copy of this licence, visit http://creativecommons.org/licenses/by/4.0/. The Creative Commons Public Domain Dedication waiver (http://creativecommons.org/publicdomain/zero/1.0/) applies to the data made available in this article, unless otherwise stated in a credit line to the data. 


\section{Background}

Dengue fever is the most prevalent human arbovirosis and a major public health issue in tropical and subtropical countries with epidemic outbreaks [1, 2]. Dengue viruses are subdivided in 4 serotypes (DENV-1 to 4). There is a lack of specific treatments, vector control measures regularly fail to prevent epidemics and safe preventive dengue vaccines are not widely available [35]. While new prevention methods are being developed, clinical management strategies are of prime importance.

Dengue has a wide spectrum of clinical presentations usually starting by an abrupt onset of fever, malaise, skin rash, headache, anorexia/vomiting, diarrhea, and abdominal pain, often with unpredictable clinical evolution. Clinical outcome can vary from a self-limiting non-severe condition to a potentially lethal disease subsequent to a vascular permeability resulting in leakage of fluids into serosal cavities and shock, hemorrhages, and/or organ failures [4]. Warning signs of severe dengue include persisting vomiting, abdominal pain, lethargy/anxiety, mucosal bleeding, liquid accumulation, hepatomegaly, and rapid hematocrit increase concurrent with a platelet count drop [4].

In New Caledonia (NC), a French South Pacific Island Territory of 270,000 inhabitants, dengue is a closely monitored notifiable disease, enabling the collection of reliable documentation of dengue cases. Dengue fever outbreaks frequency is increasing in $\mathrm{NC}$, and is associated to the emergent co-circulation of several DENV serotypes and other arboviruses, i.e. chikungunya and Zika viruses [6-8]. An uninterrupted circulation of DENV-1 has been documented in NC between 2007 and 2018.

During the 2017 dengue outbreak, three serotypes have co-circulated (DENV-1, DENV-2, DENV-3) for the first time. Four thousand three hundred seventy-nine dengue cases were declared among which 2372 (54\%) were biologically confirmed by RT-qPCR [6]. Fifteen patients died (lethality rate $=0.3 \%$ ). The hospitalization rate was exceptionally high $(11.5 \%$ versus $3.5 \%$ during the 2012-2013 outbreak, 2.1\% during the 2008-2009 outbreak, and 4.5\% during the 2003 outbreak).

Identifying risk factors for severe dengue is of prime importance to improve patients' medical care and better manage in-hospital patient flow. Such risk factors may differ depending on the region of the world considered, in link with populations' genetics and way of living. To our knowledge, risk factors for severe dengue have been mostly explored in countries where dengue is endemic and have never been explored in the Pacific region, where dengue has an epidemic mode of circulation. Assessing the reliability of identified risk factor for severe dengue in epidemiological contexts where dengue has an epidemic mode of circulation is important to relieve the health care system upon outbreaks.
Furthermore, the expansion of dengue in more temperate countries will certainly lead the hospitals to be overwhelmed, and operational tools developed in epidemic countries would help better manage in-hospital patient flow.

In 2014-2015, the Pacific region and South America experienced a Zika pandemic. This pandemic occurred in countries where dengue circulates actively, it is therefore crucial to determine whether a previous Zika infection represents a risk factor for severe dengue.

The purposes of this study were to investigate clinical and biological parameters associated with severe dengue and elaborate an operational model to score patients' risk to develop severe dengue in the $\mathrm{NC}$ medical facilities setting.

\section{Methods}

\section{Study population}

A total of 416 patients were admitted to the Territorial Hospital of New Caledonia between January 1st 2017 and July 31st 2017 with a diagnosis of dengue fever. Among them, 385 were biologically confirmed using RT-qPCR [9], of which 383 were enrolled in this study (Fig. 1).

\section{Data collection}

Patients' clinical and biological characteristics were retrieved from hospital medical records (DxCareMedasys), dengue notification sheets and completed by a telephone interview using a standardized questionnaire. Although sometimes contested in the litterature, we

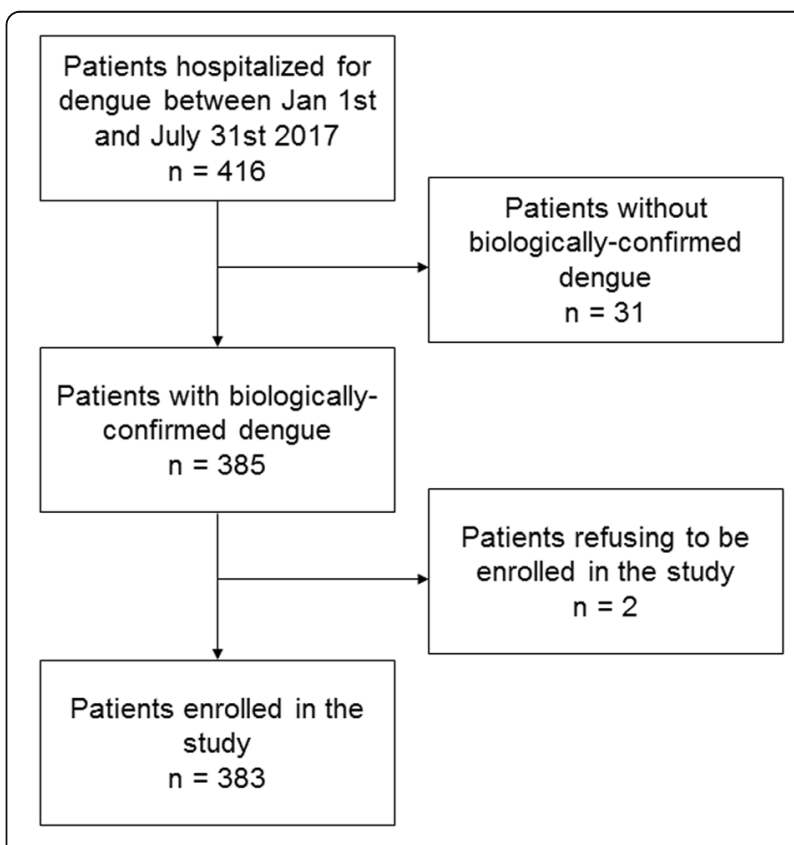

Fig. 1 STROBE flowchart describing patients enrolment in the study 
opted for a binary gender classification. No patient enrolled expressed difficulty with this classification. Collected data included patients' gender, age, ethnicity, medical history, treatments, substance abuse (tobacco, alcohol $>3$ units/day, cannabis, kava), clinical and biological parameters, presence of warning and severe signs, infecting dengue serotype and previous dengue and Zika infection. Dengue serotyping by RT-qPCR and IgG serology for dengue $\left(\mathrm{PanBio}^{\circ}\right)$ and Zika (Euroimmun ${ }^{\circ}$ ) were performed.

\section{Patients classification}

Disease severity was assessed according to the WHO 2009 criteria [4]. Patients were classified as severe when they displayed at least one of the following criteria: severe plasma leakage (shock, liquid accumulation visualized by sonogram or $\mathrm{x}$-ray) with respiratory distress; severe hemorrhage; and/or severe organ failure (kidney, central nervous system, liver, heart). Thrombocytopenia with platelet count under $10 \times 10^{9} / \mathrm{L}$ (reference range $150-400 \times 10^{9} / \mathrm{L}$ ) associated to minor bleeding was used as an additional severity criterion. Acute renal failure was defined as a Glomerular Filtration Rate by the MDRD Equation $<60 \mathrm{~mL} / \mathrm{min} / 1.73 \mathrm{~m}^{2}$ or, for patients with previous chronic renal failure, a 2 -fold increase from their baseline creatinine level. Severe hepatitis was defined by a transaminase (AST or ALT) level above $1000 \mathrm{IU} / \mathrm{L}$.

\section{Statistical analysis and predictive models construction}

For each quantitative variable, minimum, maximum, mean and median were calculated. In order to allow the implementation of univariate analyses, quantitative variables age and biological parameters were categorized into qualitative variables. Non-severe patients were compared to severe patients. In the univariate analysis, Odds Ratio (OR) and 95\% confidence interval were calculated. Test of independence $p$-values were estimated using Fisher's exact test. Differences were considered significant if $p<0.05$. Parameters for which Fisher test $p \leq 0.2$ were used to perform the multivariate analysis. Longtime conventional cutoff point in bivariate analysis to select variables to be included in a multiple regression model [10], the $p \leq 0.2$ has been further justified and strengthened recently [11]. Odds ratio were adjusted for each variable category with the value of the other variables being fixed .A predictive model for severe dengue was built using multiple logistic regression and a descending stepwise analysis. Statistics were performed using R software (version 3.5.1 (2018-07-02)).

\section{Predictive models validation}

A $\mathrm{k}$-fold cross-validation procedure $(\mathrm{k}=10)$ was used. The subsample $\mathrm{k}$ was retained as validation data and the remaining k-1 subsamples were used as training data. The cross-validation process was repeated $k$ times, each of the $\mathrm{k}$ subsamples used exactly once as the validation data. Model performance was measured using the following indicators: sensitivity, specificity, positive predictive value, negative predictive value, Yule index [12], Youden index (sensitivity + specificity - 1) [13] and the Area Under Curve (AUC) of the Receiving Operating Characteristic (ROC) curve. The $\mathrm{R}$ code used to perform the $\mathrm{k}$-fold cross-validation procedure is provided as supplementary material (Supplementary Fig. 1). Using the variable coefficients determined in the logistic regression, patients' score was expressed as $\mathrm{p}$ (probability of developing severe dengue). The decision-making threshold was defined using the ROC curve as the best combination of sensitivity and specificity.

\section{Ethics statement}

Ethical approval was granted by the Consultative Ethics Committee of $\mathrm{NC}$, and by the internal ethical review board of the Territorial Hospital. Dengue fever is a compulsory declarative disease in NC. Oral informed consent was obtained from all participating patients or their relatives retrospectively when consulted by telephone.

\section{Results}

\section{Characteristics of the studied population}

The characteristics of the 383 PCR-confirmed dengue patients included in this study are presented in Table 1 . Patients were hospitalized on average on the 5th day after symptom onset (median $=4, \mathrm{IQR}=3$ ), for a median duration of 4 days $(\mathrm{IQR}=3)$. They were 174 men and 209 women (Sex-ratio $=0.83$ ) with an age ranging from birth to 96 years old (IQR $=34$, median 32 years). Symptoms and biological parameters available at hospital admission are summarized in Table 2. DENV-1 was the major serotype (80.6\%), followed by DENV-2 (15.9\%) and DENV-3 (3.6\%). According to the WHO 2009 classification, 299/383 patients (78\%) displayed at least one warning sign. Overall, 130 patients (34\%) developed severe dengue.

While 121 patients $(40 \%)$ with at least one warning sign developed severe dengue, only nine patients (10.7\%) without warning signs did so (Fig. 2). WHO classification thus yields a Positive Predictive Value of $93 \%$ and a Negative Predictive Value of $89.3 \%$ with our dataset. The Odds ratio (OR) for patients presenting at least one warning sign is 5.6 (Confidence Interval C195\% $[2.17 ; 13.3])$, with a corresponding Relative Risk of 3.8 [2.0; 7.1]. Hepatitis was the most frequent severity criterion accounting for $41.5 \%$ of severe cases. Twenty-two patients (5.7\%) developed major hemorrhaging, $47(12.3 \%)$ had deep thrombocytopenia $<10 \times 10^{9} / \mathrm{L}$ with minor bleeding, 
Table 1 Characteristics of 383 hospitalized patients during the 2017 dengue outbreak in New Caledonia and results of the univariate analysis

\begin{tabular}{|c|c|c|c|c|}
\hline Characteristics & Number (\%) & $\begin{array}{l}\text { Non severe cases (\%) } \\
n=253\end{array}$ & $\begin{array}{l}\text { Severe cases (\%) } \\
n=130\end{array}$ & $\begin{array}{l}\text { Odds ratio }[\mathrm{Cl} 95 \%] \\
p \text { value }\end{array}$ \\
\hline \multicolumn{5}{|l|}{ Sex } \\
\hline Men & $174(45.4 \%)$ & 109 (43.1\%) & $65(50 \%)$ & $\begin{array}{l}1.32[0.86-2.02] \\
p=0.23\end{array}$ \\
\hline Women & $209(54.6 \%)$ & $144(56.9 \%)$ & $65(50 \%)$ & Reference \\
\hline \multicolumn{5}{|l|}{ Age class } \\
\hline$<10$ years old & $48(12.4 \%)$ & $39(15.4 \%)$ & $9(6.9 \%)$ & $\begin{array}{l}0.77[0.28-2.06] \\
p=0.63\end{array}$ \\
\hline [10-20] & $69(18.0 \%)$ & $50(19.8 \%)$ & $19(14.6 \%)$ & $\begin{array}{l}1.26[0.55-2.98] \\
p=0.68\end{array}$ \\
\hline [20-30] & $72(18.8 \%)$ & $43(17 \%)$ & $29(22.3 \%)$ & $\begin{array}{l}2.22[1.01-5.11] \\
p=0.05\end{array}$ \\
\hline$[30-40]$ & $52(13.6 \%)$ & $40(15.8 \%)$ & $12(9.2 \%)$ & Reference \\
\hline$[40-50]$ & $42(11.0 \%)$ & $26(10.3 \%)$ & $16(12.3 \%)$ & $\begin{array}{l}2.03[0.83-5.11] \\
p=0.17\end{array}$ \\
\hline$[50-60]$ & $39(10.2 \%)$ & $24(9.5 \%)$ & $15(11.5 \%)$ & $\begin{array}{l}2.06[0.82-5.26] \\
p=0.16\end{array}$ \\
\hline$[60-70]$ & $24(6.3 \%)$ & $12(4.7 \%)$ & $12(9.2 \%)$ & $\begin{array}{l}3.26[1.16-9.44] \\
p=0.03\end{array}$ \\
\hline$>70$ & $37(9.7 \%)$ & 19 (7.5\%) & $18(13.8 \%)$ & $\begin{array}{l}3.10[1.25-7.97] \\
p=0.02\end{array}$ \\
\hline \multicolumn{5}{|l|}{ Self-declared ethnicity } \\
\hline Melanesian & $141(36.7 \%)$ & $92(36.4 \%)$ & $49(37.7 \%)$ & $\begin{array}{l}1.30[0.73-2.34] \\
p=0.37\end{array}$ \\
\hline European & $86(22.5 \%)$ & $61(24.1 \%)$ & $25(19.2 \%)$ & Reference \\
\hline Polynesian & $68(17.8 \%)$ & $43(17 \%)$ & $25(19.2 \%)$ & $\begin{array}{l}1.40[0.71-2.81] \\
p=0.39\end{array}$ \\
\hline Métis /Other & $63(16.4 \%)$ & $44(17.3 \%)$ & 19 (14.6\%) & $\begin{array}{l}1.05[0.51-2.15] \\
p=1\end{array}$ \\
\hline Not specified & $25(5.1 \%)$ & $12(9.2 \%)$ & $5(3.8 \%)$ & $\begin{array}{l}2.23[0.88-5.66] \\
p=0.09\end{array}$ \\
\hline \multicolumn{5}{|l|}{ Risky behavior } \\
\hline Tobacco & 105 (27.4\%) & $62(24.5 \%)$ & $43(33.1 \%)$ & $\begin{array}{l}1.52[0.95-2.42] \\
p=0.09\end{array}$ \\
\hline Cannabis & 19 (4.9\%) & $12(4.7 \%)$ & $7(5.4 \%)$ & $\begin{array}{l}1.15[0.41-2.97] \\
p=0.81\end{array}$ \\
\hline Kava ${ }^{a}$ & 15 (3.9\%) & $10(3.9 \%)$ & $5(3.8 \%)$ & $\begin{array}{l}0.98[0.29-2.89] \\
p=1\end{array}$ \\
\hline Alcohol (> 3 units/day) & $9(2.3 \%)$ & $4(1.6 \%)$ & $5(3.8 \%)$ & $\begin{array}{l}2.46[0.62-10.56] \\
p=0.17\end{array}$ \\
\hline \multicolumn{5}{|l|}{ Comorbidities } \\
\hline Obesity & 95 (24.8\%) & $52(20.6 \%)$ & $43(33.1 \%)$ & $\begin{array}{l}1.91[1.18-3.07] \\
p=0.08\end{array}$ \\
\hline Diabetes & $34(8.8 \%)$ & $20(7.9 \%)$ & $14(10.8 \%)$ & $\begin{array}{l}1.41[0.67-2.89] \\
p=0.35\end{array}$ \\
\hline Dyslipidemia & $27(7.0 \%)$ & $13(5.1 \%)$ & $14(10.8 \%)$ & $\begin{array}{l}2.22[1.0-4.97] \\
p=0.06\end{array}$ \\
\hline Hypertension & $66(17.2 \%)$ & $31(12.3 \%)$ & $35(26.9 \%)$ & $\begin{array}{l}2.63[1.53-4.54] \\
p<0.01\end{array}$ \\
\hline Heart disease & $21(5.5 \%)$ & $11(4.3 \%)$ & $10(7.7 \%)$ & $\begin{array}{l}1.83[0.74-4.51] \\
p=0.23\end{array}$ \\
\hline
\end{tabular}


Table 1 Characteristics of 383 hospitalized patients during the 2017 dengue outbreak in New Caledonia and results of the univariate analysis (Continued)

\begin{tabular}{|c|c|c|c|c|}
\hline Characteristics & Number (\%) & $\begin{array}{l}\text { Non severe cases (\%) } \\
n=253\end{array}$ & $\begin{array}{l}\text { Severe cases (\%) } \\
n=130\end{array}$ & $\begin{array}{l}\text { Odds ratio }[\mathrm{Cl} 95 \%] \\
p \text { value }\end{array}$ \\
\hline Lung diseases & $31(8.1 \%)$ & $24(9.5 \%)$ & $7(5.4 \%)$ & $\begin{array}{l}0.55[0.21-1.26] \\
p=0.23\end{array}$ \\
\hline Renal failure & $9(2.3 \%)$ & $4(1.6 \%)$ & $5(3.8 \%)$ & $\begin{array}{l}2.46[0.62-10.56] \\
p=0.17\end{array}$ \\
\hline Immunodepression & $7(1.8 \%)$ & $5(2 \%)$ & $2(1.5 \%)$ & $\begin{array}{l}0.81[0.10-3.99] \\
p=1\end{array}$ \\
\hline Cancer & $14(3.7 \%)$ & $8(3.2 \%)$ & $6(4.6 \%)$ & $\begin{array}{l}1.49[0.47-4.46] \\
p=0.57\end{array}$ \\
\hline Risk of bleeding ${ }^{b}$ & $9(2.3 \%)$ & $6(2.4 \%)$ & $3(2.3 \%)$ & $\begin{array}{l}1.0[0.20-3.97] \\
p=1\end{array}$ \\
\hline \multicolumn{5}{|l|}{ Number of clinical problems } \\
\hline No medical history & $201(52.5 \%)$ & $142(56.1 \%)$ & $59(45.4 \%)$ & Reference \\
\hline 1 & $87(22.7 \%)$ & $59(23.3 \%)$ & $28(21.5 \%)$ & $\begin{array}{l}1.04[0.58-1.80] \\
p=1\end{array}$ \\
\hline 2 or more & $95(24.8 \%)$ & $52(20.6 \%)$ & $43(33.1 \%)$ & $\begin{array}{l}2.12[1.29-3.49] \\
p<0.01\end{array}$ \\
\hline \multicolumn{5}{|l|}{ History of arbovirus infection } \\
\hline Presence of dengue $\lg G$ & $132(34.5 \%)$ & $68(26.9 \%)$ & $64(49.2 \%)$ & $\begin{array}{l}2.93[1.83-4.75] \\
p<0.001\end{array}$ \\
\hline Presence of Zika lgG & $42(11.0 \%)$ & $25(9.9 \%)$ & $17(13.1 \%)$ & $\begin{array}{l}1.30[0.66-2.53] \\
p=0.49\end{array}$ \\
\hline \multicolumn{5}{|l|}{ Treatment } \\
\hline Paracetamol & $300(78.3 \%)$ & $203(80.2 \%)$ & $97(74.6 \%)$ & $\begin{array}{l}0.72[0.44-1.20] \\
p=0.24\end{array}$ \\
\hline Corticosteroids & $2(0.5 \%)$ & 0 & $2(1.5 \%)$ & - \\
\hline NSAl & $6(1.6 \%)$ & $4(1.6 \%)$ & $2(1.5 \%)$ & $\begin{array}{l}1.00[0.12-5.56] \\
p=1\end{array}$ \\
\hline Anticoagulants & $9(2.3 \%)$ & $3(1.2 \%)$ & $6(4.62 \%)$ & $\begin{array}{l}3.92[0.98-19.96] \\
p=0.07\end{array}$ \\
\hline PAl & $38(9.9 \%)$ & $19(7.5 \%)$ & 19 (14.6\%) & $\begin{array}{l}2.10[1.06-4.17] \\
p=0.03\end{array}$ \\
\hline Traditional medicine & $71(18.5 \%)$ & $46(18.2 \%)$ & $25(19.2 \%)$ & $\begin{array}{l}1.07[0.62-1.83] \\
p=0.89\end{array}$ \\
\hline \multicolumn{5}{|l|}{ Symptoms } \\
\hline Fever & $347(90.6 \%)$ & $231(91.3 \%)$ & $116(89.2 \%)$ & $\begin{array}{l}0.79[0.39-1.64] \\
p=0.58\end{array}$ \\
\hline Muscle soreness/myalgia & $249(65.0 \%)$ & $161(63.6 \%)$ & $88(67.7 \%)$ & $\begin{array}{l}1.20[0.77-1.88] \\
p=0.50\end{array}$ \\
\hline Arthralgia & $174(45.4 \%)$ & $110(43.5 \%)$ & $64(49.2 \%)$ & $\begin{array}{l}1.26[0.82-1.93] \\
p=0.33\end{array}$ \\
\hline Headaches & $261(68.1 \%)$ & $175(69.2 \%)$ & $86(66.2 \%)$ & $\begin{array}{l}0.87[0.56-1.37] \\
p=0.56\end{array}$ \\
\hline Retro-orbital pain & $98(25.6 \%)$ & $71(28.1 \%)$ & $27(20.8 \%)$ & $\begin{array}{l}0.67[0.40-1.11] \\
p=0.14\end{array}$ \\
\hline Diarrhea & $129(33.7 \%)$ & $85(33.6 \%)$ & $44(33.8 \%)$ & $\begin{array}{l}1.01[0.64-1.58] \\
p=1.0\end{array}$ \\
\hline Nausea/vomiting & $209(54.6 \%)$ & $135(53.4 \%)$ & $74(56.9 \%)$ & $\begin{array}{l}1.15[0.75-1.77] \\
p=0.52\end{array}$ \\
\hline Skin rash & $130(33.9 \%)$ & $95(37.5 \%)$ & $35(26.9 \%)$ & $\begin{array}{l}0.61[0.38-0.97] \\
p=0.041\end{array}$ \\
\hline
\end{tabular}


Table 1 Characteristics of 383 hospitalized patients during the 2017 dengue outbreak in New Caledonia and results of the univariate analysis (Continued)

\begin{tabular}{|c|c|c|c|c|}
\hline Characteristics & Number (\%) & $\begin{array}{l}\text { Non severe cases (\%) } \\
n=253\end{array}$ & $\begin{array}{l}\text { Severe cases }(\%) \\
n=130\end{array}$ & $\begin{array}{l}\text { Odds ratio }[\mathrm{Cl} 95 \%] \\
p \text { value }\end{array}$ \\
\hline Conjunctival hyperemia & $41(10.7 \%)$ & $33(13.0 \%)$ & $8(6.2 \%)$ & $\begin{array}{l}0.44[0.18-0.95] \\
p=0.053\end{array}$ \\
\hline Edema & $18(4.7 \%)$ & $8(3.2 \%)$ & $10(7.7 \%)$ & $\begin{array}{l}2.54[0.96-6.90] \\
p=0.071\end{array}$ \\
\hline Gingivorrhagia & $58(15.1 \%)$ & $33(13.0 \%)$ & $25(19.2 \%)$ & $\begin{array}{l}1.59[0.89-2.80] \\
p=0.13\end{array}$ \\
\hline Purpura & $78(20.4 \%)$ & $47(18.6 \%)$ & $31(23.8 \%)$ & $\begin{array}{l}1.37[0.81-2.29] \\
p=0.23\end{array}$ \\
\hline Epistaxis & $58(15.1 \%)$ & $34(13.4 \%)$ & $24(18.5 \%)$ & $\begin{array}{l}1.46[0.81-2.58] \\
p=0.23\end{array}$ \\
\hline Hematuria/blood in stools & $19(5.0 \%)$ & $6(2.4 \%)$ & $13(10 \%)$ & $\begin{array}{l}4.49[1.71-13.30] \\
p<0.01\end{array}$ \\
\hline \multicolumn{5}{|l|}{ Alert signs } \\
\hline Abdominal pain & $147(38.4 \%)$ & $88(34.8 \%)$ & $59(45.4 \%)$ & $\begin{array}{l}1.56[1.01-2.40] \\
p=0.046\end{array}$ \\
\hline Persistant vomiting & $42(11.0 \%)$ & $28(11.1 \%)$ & $14(10.7 \%)$ & $\begin{array}{l}0.97[0.48-1.90] \\
p=1.0\end{array}$ \\
\hline Clinical liquid accumulation & $28(7.3 \%)$ & $11(4.3 \%)$ & $17(13.1 \%)$ & $\begin{array}{l}3.28[1.50-7.50] \\
p<0.01\end{array}$ \\
\hline Mucosal bleeding & $170(44.4 \%)$ & $83(32.8 \%)$ & $87(66.9 \%)$ & $\begin{array}{l}4.12[2.64-6.51] \\
p<0.01\end{array}$ \\
\hline Lethargy/anxiety & $72(18.8 \%)$ & $46(18.2 \%)$ & $26(20.0 \%)$ & $\begin{array}{l}1.13[0.65-1.92] \\
p=0.68\end{array}$ \\
\hline Hepatomegaly & $17(4.4 \%)$ & $9(3.6 \%)$ & $8(6.2 \%)$ & $\begin{array}{l}1.78[0.64-4.83] \\
p=0.30\end{array}$ \\
\hline Increase in $\mathrm{Ht}$ and platelet count drop & $68(17.8 \%)$ & $37(14.6 \%)$ & $31(23.8 \%)$ & $\begin{array}{l}1.83[1.07-3.12] \\
p=0.034\end{array}$ \\
\hline \multicolumn{5}{|l|}{ Biological parameters } \\
\hline Normal platelet count & $225(58.7 \%)$ & $175(69.1 \%)$ & $50(38.4 \%)$ & Reference \\
\hline Platelets $<30 \times 10^{9} / \mathrm{L}$ & $134(35.0 \%)$ & $59(23.3 \%)$ & 75 (57.7\%) & $\begin{array}{l}4.42[2.79-7.08] \\
p<0.01\end{array}$ \\
\hline $\mathrm{GFR}<60 \mathrm{~mL} / \mathrm{min}$ & $42(11.0 \%)$ & $8(3.1 \%)$ & $34(26.1 \%)$ & $\begin{array}{l}9.28[4.22-22.90] \\
p<0.01\end{array}$ \\
\hline Normal AST & $217(56.7 \%)$ & 157 (62\%) & $60(46.1 \%)$ & Reference \\
\hline AST $>10 N$ & $110(28.7 \%)$ & $51(20 \%)$ & 59 (45.4\%) & $\begin{array}{l}3.01[1.87-4.89] \\
p<0.01\end{array}$ \\
\hline Normal ALT & $275(71.8 \%)$ & $198(78.3 \%)$ & 77 (59.2\%) & Reference \\
\hline $\mathrm{ALT}>10 \mathrm{~N}$ & $51(13.3 \%)$ & 10 (3.9\%) & $41(31.5 \%)$ & $\begin{array}{l}10.34[5.10-22.94] \\
p<0.01\end{array}$ \\
\hline
\end{tabular}

PAI Platelet aggregation inhibitor, NSAI Non-steroidal anti-inflammatory, GFR Glomerular filtration rate, AST Aspartate AminoTransferase, ALT Alanine AminoTransferase

${ }^{a}$ Kava: traditional beverage produced from poivrier roots, consumed throughout the cultures of Polynesia, Melanesia, and parts of Micronesia for its sedating and euphoriant effect

${ }^{\mathrm{b}}$ Risk of bleeding refers to comorbidities with previous risk of hemorrhage (menorrhagia, endometriosis, adenomyosis, gastric ulcer, immunological thrombopenic purpura)

and 26 (6.8\%) developed a shock, corresponding to $16.9,36.2$ and $20 \%$ of severe cases, respectively (Fig. 3). A total of 182 (47.5\%) patients presented comorbidities. The most frequent comorbidity was obesity $(24.8 \%)$, accounting for $33.1 \%$ of severe cases
(Table 1). Thirteen patients died (lethality rate $=$ 3.4\%): ten during hospitalization and three after discharge. In-hospital deaths involved $7 / 10$ patients younger than 55 years old without notable medical history. Previous dengue infection was recorded in 7/ 
Table 2 Clinical and biological parameters at hospital admission in the 383 hospitalized patients

\begin{tabular}{|c|c|}
\hline Clinical parameters & Number (\%) \\
\hline \multicolumn{2}{|l|}{ Symptom } \\
\hline Fever & 347 (90.6\%) \\
\hline Muscle soreness/myalgia & $249(65.0 \%)$ \\
\hline Arthralgia & $174(45.4 \%)$ \\
\hline Headache & $261(68.1 \%)$ \\
\hline Retro-orbital pain & $98(25.6 \%)$ \\
\hline Diarrhea & 129 (33.7\%) \\
\hline Nausea/vomiting & $209(54.6 \%)$ \\
\hline Skin rash & $130(33.9 \%)$ \\
\hline Conjunctival hyperemia & $41(10.7 \%)$ \\
\hline Edema & $18(4.7 \%)$ \\
\hline Gingivorrhagia & $58(15.1 \%)$ \\
\hline Purpura & 78 (20.4\%) \\
\hline Epistaxis & $58(15.1 \%)$ \\
\hline Hematuria/blood in stools & 19 (5.0\%) \\
\hline Shock syndrome & $26(6.8 \%)$ \\
\hline Major bleeding & $47(12.3 \%)$ \\
\hline \multicolumn{2}{|l|}{ Alert signs } \\
\hline Abdominal pain & $147(38.4 \%)$ \\
\hline Persistent vomiting & $42(11.0 \%)$ \\
\hline Clinical liquid accumulation & $28(7.3 \%)$ \\
\hline Mucosal bleeding & 170 (44.4\%) \\
\hline Lethargy/anxiety & $72(18.8 \%)$ \\
\hline Hepatomegaly & $17(4.4 \%)$ \\
\hline $\begin{array}{l}\text { Increase in hematocrit + drop in } \\
\text { platelets count }\end{array}$ & $68(17.8 \%)$ \\
\hline Biological parameters & $\begin{array}{l}\text { Median [min; } \max \text { ( } \% \text { of } \\
\text { available results) }\end{array}$ \\
\hline Platelets $\left(10^{9} / \mathrm{L}\right)$ & $48[3 ; 360](93.7)$ \\
\hline Hemoglobin (g/dL) & $14[6 ; 22](94.5)$ \\
\hline Hematocrit (\%) & $41[16 ; 61](94)$ \\
\hline Neutrophils $\left(/ \mathrm{mm}^{3}\right)$ & 1895 [320; 19020] (92) \\
\hline Lymphocytes $\left(/ \mathrm{mm}^{3}\right)$ & $1535[140 ; 8940](90.8)$ \\
\hline Albuminaemia (g/L) & $36[19 ; 46](13.8)$ \\
\hline Protidaemia (g/L) & $58[24 ; 91](10.4)$ \\
\hline Urea (mmol/L) & $4[0 ; 41](76)$ \\
\hline Creatinin $(\mu \mathrm{mol} / \mathrm{L})$ & $71[18 ; 927](78.3)$ \\
\hline AST (IU/L) & $184[17 ; 10336](85)$ \\
\hline ALT (IU/L) & $116[9 ; 8040](85)$ \\
\hline CPK & 305 [3-74063] (28) \\
\hline Lipase (IU/L) & 53 [9; 2707] (33.4) \\
\hline CRP (mg/L) & $14[0 ; 327](56.6)$ \\
\hline
\end{tabular}

8 patients who died within a week; all three circulating serotypes were involved, including one case of coinfection.

\section{Factors associated with severe dengue in univariate analysis}

Factors highly associated with severe dengue in univariate analysis included age ]20-30] years old and ]60-70] years old (OR 2.22 CI95\% [1.01-5.11] and 3.26 CI95\% [1.16-9.44]), comorbidities (hypertension OR 2.63 CI95\% [1.53-4.54], obesity OR 1.91 CI95\% [1.18-3.07], dyslipidaemia OR 2.22 CI95\% [1.0-4.97] and more than two comorbidities OR 1.98 CI95\% [1.29-3.49]), previous dengue infection (OR 2.93 CI95\% [1.83-4.75]), use of platelet aggregation inhibitors (OR 2.1 CI95\% [1.064.17]), presence of at least one alert sign (OR 5.6 CI95\% [2.17-13.3]), platelets <30 $<10^{9} / \mathrm{L} \quad$ (OR 4.42 CI95\% [2.79-7.08]), Glomerular Filtration Rate $<60 \mathrm{~mL} / \mathrm{min}$ (OR 9.28 CI95\% [4.22-22.90]), AST $>10 \mathrm{~N}$ (OR 3.01 CI95\% [1.87-4.89]) and ALT > $10 \mathrm{~N}$ (OR 10.34 CI95\% [5.10-22.94]) (Table 1).

\section{Multivariate analysis and construction of the predictive models}

Variables significantly associated with dengue severity in univariate analysis with $p \leq 0.2$ and available at hospital admission were taken into account in the multivariate analysis. These variables were: age, comorbidities (hypertension, myocardiopathy, dyslipidemia, obesity), excessive tobacco and alcohol consumption, anticoagulant or use of platelet aggregation inhibitors, mucosal bleeding, hematuria and/or presence of blood in stools, skin rash, clinical liquid accumulation, abdominal pain, simultaneous hematocrit increase and platelet count drop, platelets $<30 \times 10^{9} / \mathrm{L}$, ALT and/or AST $>10 \mathrm{~N}$. Raw analysis of the dataset showed that certain age groups in females were more at risk to develop severe dengue, while no difference was observed between age groups in men. This suggested the existence of an interaction between age and sex. This interaction was confirmed using a bivariate analysis taking age and sex as variables. Multivariate analysis further confirmed this interaction between age and sex: women between 20 and 30 years and men over 60 years were more at risk for severe dengue (OR 7.79 CI95\% [1.87-41.86] and 8.45 CI95\% [1.5953.3], respectively). An analysis for each modality of the variable sex was thus performed and two different predictive models were built according to sex.

Descending stepwise analysis identified the best explanatory variables for progression towards severe dengue (Table 3). In the model for females, these variables were: age class, hypertension, skin rash, mucosal bleeding, platelets count $<30 \times 10^{9} / \mathrm{L}$ and ALT $>10 \mathrm{~N}$. In the model for males, these variables were: age class, 


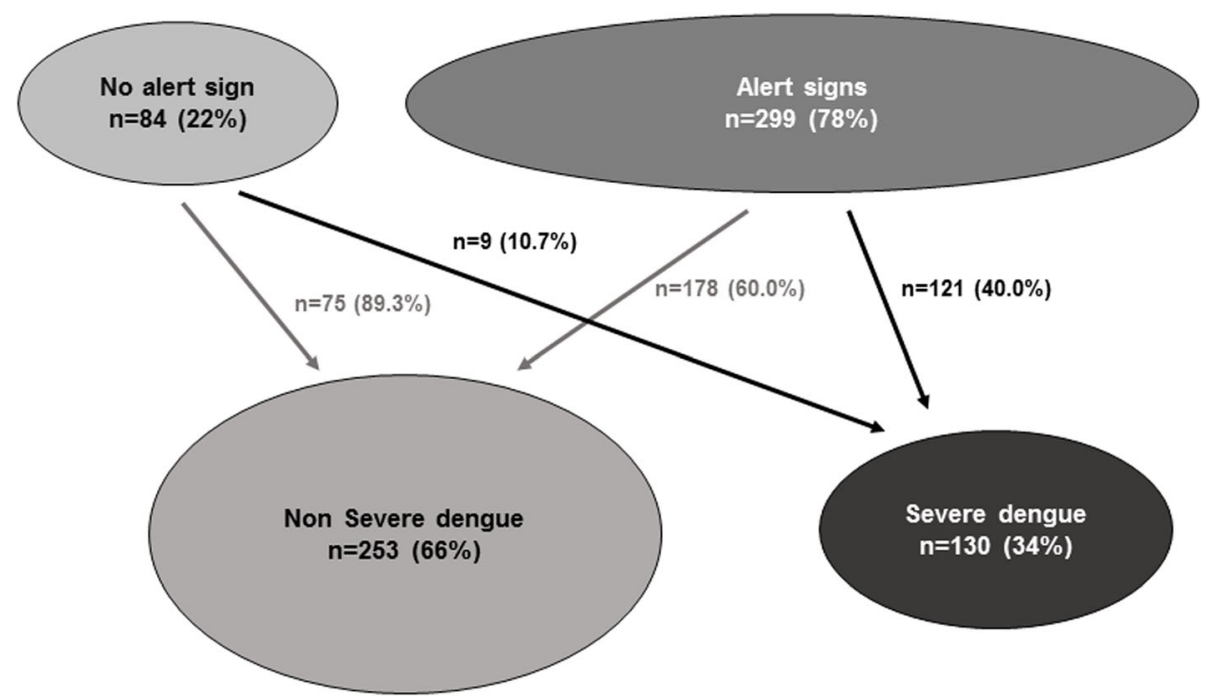

Fig. 2 Classification of the 383 hospitalized patients according to the presence of alert and severity signs (2017 dengue outbreak, New Caledonia). Scheme of dengue cases distribution, showing the percentage of cases with and without alert signs and their evolution to nonsevere and severe dengue, according to the WHO 2009 classification adapted for our study with minor modifications (thrombocytopenia < 10 $\times$ $10^{9} / \mathrm{L}$ associated to minor bleeding was used as an additional severity criterion)

excessive alcohol consumption, mucosal bleeding, platelets count $<30 \times 10^{9} / \mathrm{L}$ and ALT $>10 \mathrm{~N}$. The predictive models provide a score. Score threshold is derived from the ROC curve as the threshold optimal value for which

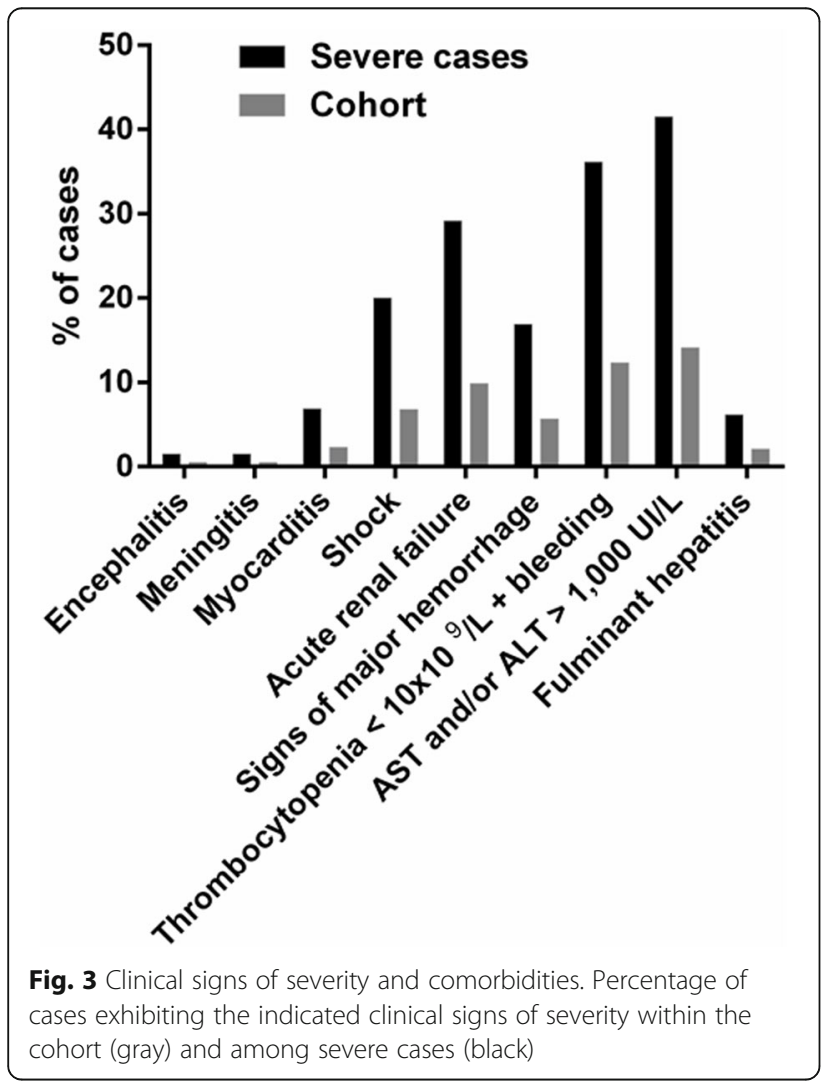

sensitivity and specificity are the highest for the whole dataset. A score $\geq 0.36$ for females and $\geq 0.34$ for males indicates a high probability to develop severe dengue. The Excel spreadsheet enabling the calculation of this risk score is presented in Supplementary Fig. 2. Examples of models' usage are presented in Supplementary Fig. 3.

\section{Performance of the models}

A $\mathrm{k}$-fold cross-validation procedure $(\mathrm{k}=10)$ showed that both models for females and males were robust and efficient (Fig. 4), yielding a median AUC of 0.80 (Interquartile Range IQR $=0.08$, range [0.638; 0.952]) and 0.88 (IQR $=0.13$, range $[0.701 ; 1.00]$ ), respectively and a high median Negative Predictive Value (92.8 and $91.3 \%$, respectively). ROC curves obtained for the 10 replicates of the $\mathrm{k}$-fold cross-validation procedure for females and males are shown in Supplementary Figs. 4 and 5 respectively.

Models were challenged on a cohort of 130 patients (66 females, 64 males) hospitalized for dengue in NC in 2018. Contingency tables depicting the number of severe dengue cases among the 2018 dataset and the number of severe dengue retrieved from models predictions are shown in Supplementary Fig. 6. Models for females and males yielded a sensitivity of 73 and $84 \%$, a specificity of 88 and 71\%, a Positive Predictive Value of 65 and 55\%, a Negative Predictive Value of 92 and 91\%, a Youden index of 0.62 and 0.55 and a Yule Q index of 0.91 and 0.62 , respectively. 
Table 3 Results of multivariate analysis concerning determinant factors of dengue severity used to build the predictive models for females and males

\begin{tabular}{|c|c|c|c|}
\hline & Crude odds ratio & Adjusted odds ratio (females) & Adjusted odds ratio (males) \\
\hline \multicolumn{4}{|l|}{ Age class (years) } \\
\hline$\leq 10$ & $0.77[0.28-2.05]$ & $2.52[0.39-16.94]$ & $0.77[0.12-4.72]$ \\
\hline ]10-20] & $1.26[0.55-2.98]$ & $3.22[0.62-19.57]$ & $0.88[0.18-4.52]$ \\
\hline ]20-30] & $2.22[1.01-5.10]$ & $7.79[1.87-41.86]$ & $0.26[0.04-1.57]$ \\
\hline ]40-60] & $2.04[0.94-4.64]$ & $5.74[1.33-31.35]$ & $1.21[0.24-6.37]$ \\
\hline$>60$ & $3.17[1.42-7.44]$ & $3.54[0.58-24.36]$ & $8.45[1.59-53.3]$ \\
\hline Hypertension & $2.7[1.6-4.7]$ & $4.68[1.24-19.75]$ & \\
\hline Alcohol consumption & $2.46[0.62-10.56]$ & & 20.83 [1.93-807.49] \\
\hline Mucosal bleeding & $4.12[2.64-6.51]$ & $4.66[2.08-11.14]$ & $9.79[3.75-28.72]$ \\
\hline Clinical liquid accumulation & $3.28[1.50-7.50]$ & $3.88[0.87-18.19]$ & \\
\hline Skin rash & $0.61[0.38-0.97]$ & $0.41[0.16-0.97]$ & \\
\hline \multicolumn{4}{|l|}{ Platelets } \\
\hline$<30.10^{9} / \mathrm{L}$ & $4.42[2.79-7.08]$ & $2.83[1.26-6.45]$ & $5.84[2.21-17.04]$ \\
\hline \multicolumn{4}{|l|}{$A L T(I U / L)$} \\
\hline$\geq 10 \mathrm{~N}$ & 10.34 [5.10-22.94] & 14.31 [4.93-47.67] & 243.09 [28.75-6130.86] \\
\hline
\end{tabular}

All parameters are risk factors for dengue severity albeit skin rash that appears as a protective factor to develop severe dengue in females

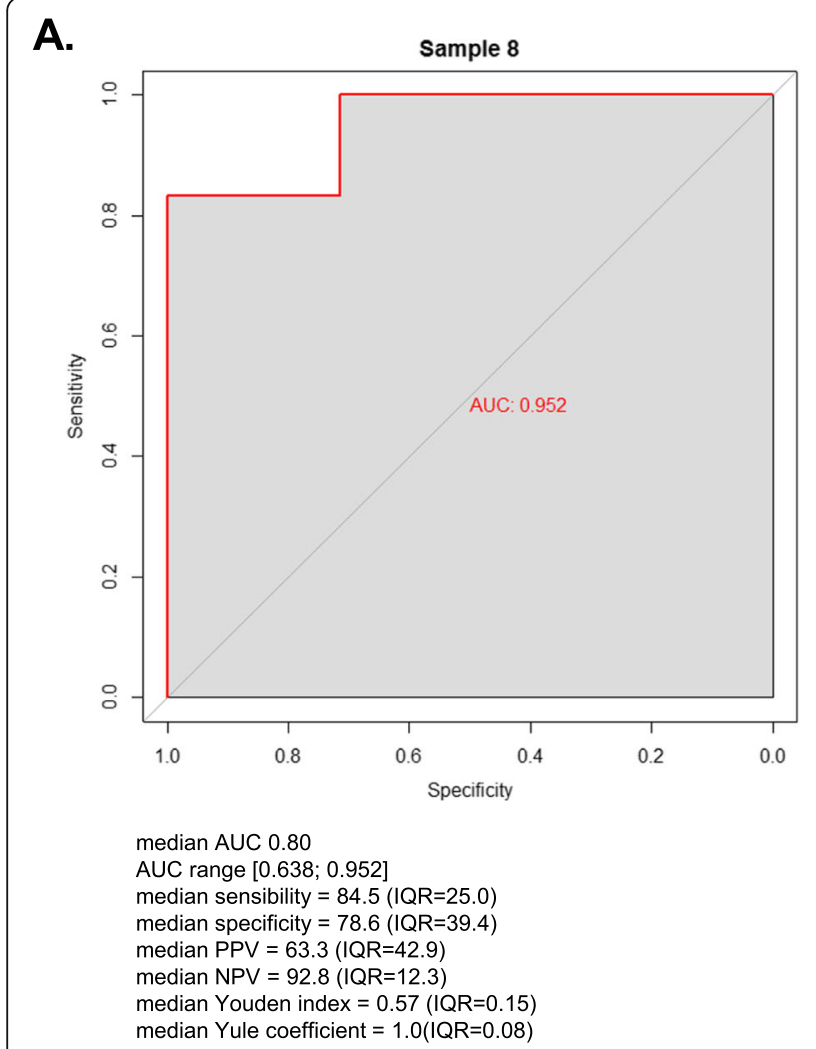

B.

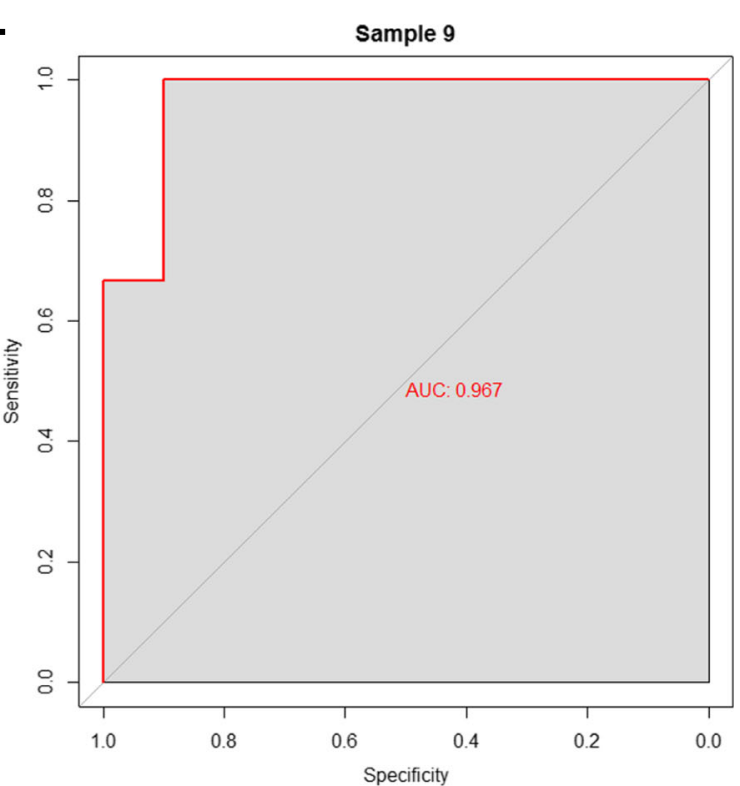

Median AUC 0.88

AUC range $[0.701 ; 1.00]$

median sensibility $=84.5(\mathrm{IQR}=28.6)$

median specificity $=95.5(\mathrm{IQR}=16.1)$

median PPV $=92.9(\mathrm{IQR}=22.3)$

median NPV $=91.3(\mathrm{IQR}=15.4)$

median Youden index $=0.74(\mathrm{IQR}=0.19)$

median Yule coefficient $=1(\mathrm{IQR}=0.0)$

Fig. 4 Performance of predictive models for severe dengue according to the sex, New Caledonia 2017. Receiving Operating Characteristic (ROC) curves for the best model for females (a) and the best model for males (b). Median AUC, Sensitivity, Specificity, Positive Predictive Value (PPV), Negative Predictive Value (NPV), Youden index and Yule Q coefficient are indicated for each model 


\section{Discussion}

The 2017 dengue outbreak in New Caledonia was characterized by a high hospitalization rate leading to an overwhelming of emergency rooms and hospitalization units. Clinicians and epidemiologists expressed the necessity to develop a comprehensive operational tool in order to improve medical care and in-patients flow in local hospitals. Based on a detailed analysis of 383 hospitalized patients, we identified important demographical, clinical and biological parameters associated with severe dengue. Parameters easily available in routine practice were used to score patients' risk of developing severe dengue.

Importantly, we confirm that the WHO 2009 criteria to evaluate the severity of dengue infection are applicable in NC, with a Positive Predictive Value for the presence of at least one warning sign of $93 \%$ and a Negative Predictive Value (NPV) of $89.3 \%$ on our 2017 dataset. The NPV yielded by our models challenged for the management of the 2018 dengue outbreak in NC were similar, reaching 92 and $91 \%$ for females and males respectively, consolidating the reliability of our models. Most importantly, while WHO warning signs are serious symptoms resulting from an already advanced dengue infection and relying on clinicians' interpretation, the criteria used by our model are mostly objective and available early in the development of dengue. The criteria of our models indeed rely on demographic (age), behavioral (alcohol consumption) or biomedical (AST, platelet count) data or data linked to a preexisting medical condition (hypertension), which are available early in the development of dengue, as soon as hospital admission. These criteria therefore allow an earlier and reliable assessment of the risk of developing severe dengue.

Most of the risk factors we identified in univariate analysis have been described in other studies, i.e. age [14, 15], comorbidities such as hypertension [16] or diabetes $[14,16,17]$, persistent vomiting $[15,18]$, increase in hematocrit $[14,18]$. A platelet count $<30 \times 10^{9} / \mathrm{L}$ was also strongly associated with severe dengue in our study (OR 4.42) like in other studies [14, 18, 19]. However, no consensus on severe thrombocytopenia definition was obtained in a recent working group of dengue researchers and public health specialists to develop standardized endpoints, and they remained divided on whether a rapid decreasing trend or a specific platelet count should be case-defining [20]. Factors not associated with severity in $\mathrm{NC}$ are sex, ethnicity and substance abuse. In our study, the detected serotype responsible for the acute infection is not linked to the severity of the disease, unlike previous findings [21].

Dengue disease can have a greater impact in case of comorbidity. Our multivariate analysis identifies hypertension as a risk factor for severe dengue among females. Others have identified chronic comorbidities such as asthma, obesity, diabetes, hypertension and heart diseases to yield ORs of severe dengue diseases of about 2 to 4 in infected patients [22]. However, the link between comorbidity and severe dengue may be due to confounding factors: Comorbidities are more frequent among more aged persons in the population. Further, as people age, their probability of having undergone several dengue infections with successive serotypes during their lifetime is higher, leading to a greater risk of secondary infection.

We report a 4.7 fold increased risk of severe dengue in patients who had a serologically documented history of previous dengue infection, in accordance with the Antibody-Dependent Enhancement (ADE) theory stating that secondary dengue infections are usually more severe [23]. However, as dengue serology is usually not available at hospital admission, the presence of dengue IgG antibodies was not included in our multivariate analysis. For the first time to our knowledge, we have investigated the influence of a previous Zika infection on dengue outcome, and we found no association with dengue severity. As DENV and ZIKV serological tests display partial cross-reactivity [24], the impact of previous DENV and ZIKV infection on dengue severity should be confirmed by seroneutralization tests.

We were able to build two robust and efficient logistic regression models to evaluate patients' risk of developing severe dengue in men and women. These models enable the calculation of a risk score based on simple parameters and may represent easy-to-use operational tools to help clinicians in hospitalization decision and improve in-hospital patient flux (Supplementary Figs. 2 \& 3). Different predictive models have been proposed in previous studies, based on multivariate logistic regression [25-27] or on classification and regression trees [28, 29]. Parameters used in these models were mainly age, leukocytosis and platelet count. Interestingly, Nguyen et al. developed a prognosis model taking into account vomiting, platelet count $\left(<10 \times 10^{9} / \mathrm{L}\right)$, AST level (2-fold increase) and NS1 rapid test status [27]. The model they propose yielded a very good discriminative ability (AUC 0.95), which is close to the AUC of 0.80 and 0.88 yielded by our models for females and males, respectively. However, a major caveat to their study is the absence of model cross-validation.

The bedside scoring tool we propose is very simple and easy to use, results ranging from 0 (minimal risk of severe dengue) to 1 (maximal risk). When a patient refers to the hospital with probable or confirmed dengue fever, we calculate his severity score at first hospital referral, as soon as platelet count and ALT quantification are available. If it is $\leq 0.34$ for a man and $\leq 0.36$ for a woman, we recommend medical monitoring, preferentially in a medical unit whenever possible. If patients are discharged, we invite them to be reassessed by a physician at day 4-6 post-symptoms' onset. If the score is 
greater, we propose an admission for IV fluid treatment and bio-clinical monitoring. If the score is $>0.6$ the patient should be closely monitored and if it is $>0.8$ he might require intensive care. We also systematically recommend ICU admission when platelet count is $<10 \times$ $10^{9} / \mathrm{L}$ or transaminases $>2000 \mathrm{IU} / \mathrm{L}$.

Although based on a well-documented and validated database, biological data were heterogeneous as collected at different time points after symptoms' onset, but this limit is inherent to retrospective studies. As our studied population was composed of relatively old patients (which is a characteristic of dengue epidemiology in NC), hospitalized in a single hospital (albeit the largest in NC), our models may not be valid to predict patients' risk to develop severe dengue in other populations. However, our models were prospectively validated on 130 patients during 2018 DENV-2 outbreak in NC, yielding a high Negative Predictive Value of 92 and 91\% for females and males respectively. Our models could thus be relevant for dengue severity prediction regardless of the serotype.

\section{Conclusions}

We developed a bedside score to predict dengue severity. We propose this bedside score to be deployed, tested and validated in other countries with similar dengue epidemiology, in order to optimize patients' triage, inhospital patients flux, and improve personalized medical care, thus benefiting both health practitioners and populations facing dengue outbreaks of enhanced severity.

\section{Abbreviations}

ADE: Antibody-Dependent Enhancement; ALT: Alanine Aminotransferase; AST: Aspartate Aminotransferase; AUC: Area Under the Curve; DENV: Dengue virus; ICU: Intensive Care Unit; IgG: Type G Immunoglobulin; IQR: Interquartile Range; IV: Intravenous; IU/L: International Units/L; NC: New Caledonia; NS1: Non-Structural protein 1; OR: Odds Ratio; ROC curve: Receiving Operating Characteristic curve; RT-qPCR: Reverse Transcription-quantitative Polymerase Chain Reaction; WHO: World Health Organization; ZIKV: Zika virus

\section{Supplementary Information}

The online version contains supplementary material available at https://doi. org/10.1186/s12879-021-06146-z.

Additional file 1: S1 Fig. $R$ code enabling the implementation of the $k$ fold cross-validation procedure.

Additional file 2: S2 Fig. Excel spreadsheet enabling the calculation of a bedside score predictive of severe dengue. In the operating tool, scores derived from the logistic regression models can be calculated using an Excel spreadsheet by inserting 1 if the characteristic is present. A score $\geq$ 0.36 for females and 0.34 for males indicates a high probability to develop severe dengue. The hospitalization decision is made according to the medical opinion. During hospitalization, patients are submitted to close surveillance, hyperhydration, symptomatic treatments and sometimes blood support and resuscitation measures.

Additional file 3: S3 Fig. Examples of scoring to estimate the risk to develop severe dengue using data available at the moment of hospital admission decision. In the upper example, the score of the female patient is above 0.36 , indicating a high risk to develop severe dengue. In the lower example, the score of the male patient is below 0.34 indicating a low probability to develop severe dengue.

Additional file 4: S4 Fig. Receiving operating curves obtained for females in the k-fold cross-validation procedure.

Additional file 5: S5 Fig. Receiving operating curves obtained for males in the k-fold cross-validation procedure.

Additional file 6: S6 Fig. Contingency tables showing the performance of the models for the prediction of dengue severity on dengue 2018 outbreak in New Caledonia. Absolute numbers of severe dengue observed in the dataset and predicted by the models are shown for the model for females (upper table) and the model for males (lower table).

\section{Acknowledgements}

We thank Sébastien Mabon, Sophie Lafleur, Pascal André, and Mathieu Serié from CHT Noumea for their help during this study, Antoine Biron, MarieAmélie Goujart, Nathalie Amedeo, Erwan Choblet, Gauthier Delvallez from CHT Laboratory. We thank Ludovic Floury, Viktoria Taofifenua, Anne Pfannstiel from DASS-NC, Morgan Mangeas and Magali Teurali (IRD) for their help in statistics. We dedicate this work to our dear colleague Dr Elodie Descloux who was deeply involved in arboviruses research in New Caledonia.

\section{Authors' contributions}

$E D, A V$ and CF conceived the study, IM, EK-F, CC, AM, ED recruited the patients. A-CG, EB performed the biological analyses. IM, Cl, CF, AT, ED analyzed the data, CF performed the statistical analyses, AV, DA, SL, J-PG provided access to patients' data. Cl, AT, MD-R, IM, ED and EK-F wrote the manuscript, $I M, C F, C l, E K-F$ and ED contributed equally to this work, all authors carefully revised the manuscript. All authors have read and approved the final manuscript.

\section{Funding}

This work was supported by the government of New Caledonia. The funder provided financial support to the study but did not participate in the design of the study, data collection, analysis, and interpretation or writing of the manuscript.

\section{Availability of data and materials}

The datasets used and/or analysed during the current study are available from the corresponding author on reasonable request.

\section{Declarations}

Ethics approval and consent to participate

Ethical approval was granted by the Consultative Ethics Committee of NC, and by the internal ethical review board of the Territorial Hospital. Dengue fever is a compulsory declarative disease in NC. The current research involves the human person with minimal risks and constraints. In order to comply with the local regulation related to this kind of research and following approval by the Consultative Ethics Committee of NC, verbal consent was obtained from all participating patients or their relatives retrospectively when consulted by telephone.

Consent for publication

Not applicable.

Competing interests

The authors declare no conflict of interest.

\section{Author details}

${ }^{1}$ Internal Medicine and Infectious Diseases Department, Territorial Hospital Center (CHT), Dumbea, New Caledonia. ${ }^{2}$ Health Authorities (DASS), Noumea, New Caledonia. ${ }^{3}$ Institut Pasteur in New Caledonia, URE Dengue and Arboviruses, Institut Pasteur International Network, Noumea, New Caledonia. ${ }^{4}$ Hôpitaux Universitaires Pitie Salpetriere-Charles Foix, Paris, France.

${ }^{5}$ Microbiology Laboratory, Territorial Hospital Center (CHT), Dumbea, New Caledonia. ${ }^{6}$ Institut Pasteur in New Caledonia, URE Epidemiology, Institut Pasteur International Network, Noumea, New Caledonia. 
Received: 22 November 2019 Accepted: 6 May 2021

Published online: 24 May 2021

\section{References}

1. Guzman MG, Gubler DJ, Izquierdo A, Martinez E, Halstead SB. Dengue infection. Nat Rev Dis Primers. 2016;2(1):16055. Epub 2016/08/19. https://doi. org/10.1038/nrdp.2016.55.

2. Bhatt S, Gething PW, Brady OJ, Messina JP, Farlow AW, Moyes CL, et al. The global distribution and burden of dengue. Nature. 2013;496(7446):504-7. Epub 2013/04/09. https://doi.org/10.1038/nature12060.

3. Sridhar S, Luedtke A, Langevin E, Zhu M, Bonaparte M, Machabert T, et al. Effect of dengue serostatus on dengue vaccine safety and efficacy. N Engl J Med. 2018:379(4):327-40. Epub 2018/06/14. https://doi.org/10.1056/ NEJMoa1800820

4. WHO. Dengue: guidelines for diagnosis, treatment, prevention and control: new edition. 2009

5. WHO. Dengue vaccine: WHO position paper - September 2018 - Note de synthèse de l'OMS sur le vaccin contre la dengue- septembre 2018. Wkly Epidemiol Rec. 2018;93(36):457-76.

6. DASS-NC. Documents, rapport, études - La situation sanitaire de la Nouvelle-Calédonie. Available at https://dass.gouv.nc/votre-sante/ documents-rapports-etudes. Accessed 19 May 2021.

7. Dupont-Rouzeyrol M, Aubry M, O'Connor O, et al. Epidemiological and molecular features of dengue virus type-1 in New Caledonia, South Pacific 2001-2013. Virol J. 2014;11(1):61. Epub 2014/04/02. https://doi.org/10.1186/1 743-422X-11-61.

8. Inizan C, Tarantola A, O'Connor O, et al. Dengue in New Caledonia: knowledge and gaps. Trop Med Infect Dis. 2019:4(2):95 Epub 2019/06/23.

9. CDC. Trioplex real-time RT-PCR assay instruction for use. 2017. [12.20.2018]. Available from: https://www.cdc.gov/zika/pdfs/trioplex-real-time-rt-pcr-assayinstructions-for-use.pdf.

10. Hosmer DJ, Lemeshow S, May S. Applied survival analysis: regression modeling of time to event data. 2nd ed. Wiley; 2008. p. 416. Available at https://www.wiley.com/en-us/Applied+Survival+Analysis\%3A+Regression+ Modeling+of+Time+to+Event+Data\%2C+2nd+Edition-p-9781118211588 Accessed 19 May 2021

11. Dunkler D, Plischke M, Leffondré K, Heinze G. Augmented backward elimination: a pragmatic and purposeful way to develop statistical models. PLoS One. 2014;9(11):e113677. Epub 2014/11/22. https://doi.org/10.1371/ journal.pone.0113677.

12. Udny Yule G. VII. On the association of attributes in statistics: with illustrations from the material of the childhood society. Philos Trans R Soc Lond A. 1900;194(252-261):257-319.

13. Youden W. Index for rating diagnostic tests. Cancer. 1950;3(1):32-5. https:// doi.org/10.1002/1097-0142(1950)3:1<32::AID-CNCR2820030106>3.0.CO;2-3.

14. Pang J, Hsu JP, Yeo TW, Leo YS, Lye DC. Diabetes, cardiac disorders and asthma as risk factors for severe organ involvement among adult dengue patients: a matched case-control study. Sci Rep. 2017;7(1):39872. Epub 2017/ 01/04. https://doi.org/10.1038/srep39872.

15. Amancio FF, Heringer TP, de Oliveira CC, et al. Clinical profiles and factors associated with death in adults with dengue admitted to intensive care units, Minas Gerais, Brazil. PLoS One. 2015;10(6):e0129046. Epub 2015/06/20. https://doi.org/10.1371/journal.pone.0129046.

16. Pang J, Salim A, Lee VJ, Hibberd ML, Chia KS, Leo YS, et al. Diabetes with hypertension as risk factors for adult dengue hemorrhagic fever in a predominantly dengue serotype 2 epidemic: a case control study. PLoS Negl Trop Dis. 2012;6(5):e1641. Epub 2012/05/09. https://doi.org/10.1371/ journal.pntd.0001641.

17. Figueiredo MA, Rodrigues LC, Barreto ML, et al. Allergies and diabetes as risk factors for dengue hemorrhagic fever: results of a case control study. PLoS Negl Trop Dis. 2010;4(6):e699. Epub 2010/06/10. https://doi.org/10.1371/ journal.pntd.0000699.

18. Leo YS, Thein TL, Fisher DA, Low JG, Oh HM, Narayanan RL, et al. Confirmed adult dengue deaths in Singapore: 5-year multi-center retrospective study. BMC Infect Dis. 2011;11(1):123. Epub 2011/05/17. https://doi.org/10.1186/14 71-2334-11-123.

19. Bouldouyre MA, Baumann F, Berlioz-Arthaud A, Chungue E, Lacassin F. Factors of severity at admission during an epidemic of dengue 1 in New Caledonia (South Pacific) in 2003. Scand J Infect Dis. 2006;38(8):675-81. Epub 2006/07/22. https://doi.org/10.1080/00365540600606432.
20. Tomashek KM, Wills B, See Lum LC, Thomas L, Durbin A, Leo YS, et al. Development of standard clinical endpoints for use in dengue interventional trials. PLoS Negl Trop Dis. 2018;12(10):e0006497. Epub 2018/ 10/05. https://doi.org/10.1371/journal.pntd.0006497.

21. Vicente CR, Herbinger KH, Fröschl G, Malta Romano C, de Souza Areias Cabidelle A, Cerutti Junior C. Serotype influences on dengue severity: a cross-sectional study on 485 confirmed dengue cases in Vitória, Brazil. BMC Infect Dis. 2016;16(1):320. Epub 2016/07/10. https://doi.org/10.1186/s12879016-1668-y.

22. Badawi A, Velummailum R, Ryoo SG, Senthinathan A, Yaghoubi S, Vasileva $D$, et al. Prevalence of chronic comorbidities in dengue fever and West Nile virus: a systematic review and meta-analysis. PLoS One. 2018;13(7):e0200200. https://doi.org/10.1371/journal.pone.0200200.

23. Guzman MG, Alvarez M, Halstead SB. Secondary infection as a risk factor for dengue hemorrhagic fever/dengue shock syndrome: an historical perspective and role of antibody-dependent enhancement of infection. Arch Virol. 2013;158(7):1445-59. Epub 2013/03/09. https://doi.org/10.1007/ s00705-013-1645-3.

24. Zaidi MB, Cedillo-Barron L, González YAME, et al. Serological tests reveal significant cross-reactive human antibody responses to Zika and dengue viruses in the Mexican population. Acta Trop. 2020;201:105201. Epub 2019/ 09/29. https://doi.org/10.1016/j.actatropica.2019.105201.

25. Carrasco LR, Leo YS, Cook AR, Lee VJ, Thein TL, Go CJ, et al. Predictive tools for severe dengue conforming to World Health Organization 2009 criteria. PLoS Negl Trop Dis. 2014;8(7):e2972. Epub 2014/07/11. https://doi.org/10.13 71/journal.pntd.0002972.

26. Lee IK, Liu JW, Chen YH, Chen YC, Tsai CY, Huang SY, et al. Development of a simple clinical risk score for early prediction of severe denque in adult patients. PLoS One. 2016;11(5):e0154772. Epub 2016/05/04. https://doi.org/1 0.1371/journal.pone.0154772

27. Nguyen MT, Ho TN, Nguyen W, Nguyen TH, Ha MT, Ta VT, et al. An evidence-based algorithm for early prognosis of severe dengue in the outpatient setting. Clin Infect Dis. 2017;64(5):656-63. Epub 2016/12/31. https://doi.org/10.1093/cid/ciw863.

28. Potts JA, Gibbons RV, Rothman AL, Srikiatkhachorn A, Thomas SJ, Supradish $\mathrm{PO}$, et al. Prediction of dengue disease severity among pediatric Thai patients using early clinical laboratory indicators. PLoS Negl Trop Dis. 2010; 4(8):e769. Epub 2010/08/07. https://doi.org/10.1371/journal.pntd.0000769.

29. Phakhounthong K, Chaovalit P, Jittamala P, Blacksell SD, Carter MJ, Turner P, et al. Predicting the severity of dengue fever in children on admission based on clinical features and laboratory indicators: application of classification tree analysis. BMC Pediatr. 2018;18(1):109. Epub 2018/03/15. https://doi.org/10.1186/s12887-018-1078-y.

\section{Publisher's Note}

Springer Nature remains neutral with regard to jurisdictional claims in published maps and institutional affiliations.

Ready to submit your research? Choose BMC and benefit from:

- fast, convenient online submission

- thorough peer review by experienced researchers in your field

- rapid publication on acceptance

- support for research data, including large and complex data types

- gold Open Access which fosters wider collaboration and increased citations

- maximum visibility for your research: over $100 \mathrm{M}$ website views per year

At BMC, research is always in progress.

Learn more biomedcentral.com/submissions 